\title{
Attempt to explain black hole spin in X-ray binaries by new physics
}

\author{
Cosimo Bambi ${ }^{\mathrm{a}}$ \\ Department of Physics, Center for Field Theory and Particle Physics, Fudan University, Shanghai 200433, China
}

Received: 17 November 2014 / Accepted: 20 December 2014 / Published online: 22 January 2015

(c) The Author(s) 2015. This article is published with open access at Springerlink.com

\begin{abstract}
It is widely believed that the spin of black holes in X-ray binaries is mainly natal. A significant spin-up from accretion is not possible. If the secondary has a low mass, the black hole spin cannot change too much even if the black hole swallows the whole stellar companion. If the secondary has a high mass, its lifetime is too short to transfer the necessary amount of matter and spin the black hole up. However, while black holes formed from the collapse of a massive star with solar metallicity are expected to have low birth spin, current spin measurements show that some black holes in X-ray binaries are rotating very rapidly. Here we show that, if these objects are not the Kerr black holes of general relativity, the accretion of a small amount of matter $\left(\sim 2 M_{\odot}\right)$ can make them look like very fast-rotating Kerr black holes. Such a possibility is not in contradiction with any observation and it can explain current spin measurements in a very simple way.
\end{abstract}

\section{Introduction}

When a star exhausts all its nuclear fuel, it shrinks to find a new equilibrium configuration. For very massive stars, there is no known mechanism capable of balancing their own weight: these objects undergo a complete gravitational collapse and the final product is a black hole $(\mathrm{BH})$. It is thought that in our Galaxy there are about $10^{7} \mathrm{BHs}$ formed from the gravitational collapse of massive stars. Despite this huge number, we only know about 20 stellar-mass BH candidates [1]. They live in X-ray binaries and from the study of the orbital motion of the stellar companion it is possible to infer that the mass of the compact object exceeds $3 M_{\odot}$. This is the maximum mass for a neutron or a quark star [2], and therefore a compact object exceeding this limit is classified as a $\mathrm{BH}$ candidate.

In four-dimensional general relativity, an uncharged $\mathrm{BH}$ is described by the Kerr solution and it is completely specified

\footnotetext{
a e-mail: bambi@fudan.edu.cn
}

by only two parameters, corresponding to the mass $M$ and the spin angular momentum $J$ of the object. A fundamental limit for a Kerr BH is the bound $\left|a_{*}\right| \leq 1$, where $a_{*}=J / M^{2}$ is the dimensionless spin parameter. ${ }^{1}$ For $\left|a_{*}\right|>1$ there is no event horizon in the Kerr metric and the spacetime has a naked singularity [3]. If we can measure both $M$ and $a_{*}$ of a Kerr BH, we know all the properties of the spacetime geometry. The effect of the accretion disk on the background metric is indeed negligible [4]. However, it is not easy to estimate the $\mathrm{BH}$ spin: the spin has no effects in Newtonian gravity and therefore it is necessary to probe the spacetime close to the object. At present, the spin parameter has been measured only for about 10 stellar-mass BH candidates [5,6].

It is commonly thought that the spin of stellar-mass BHs in $\mathrm{X}$-ray binaries is mainly natal and that the effect of the accretion process is negligible [7] (but see Ref. [8]). The argument can be summarized as follows. Stellar-mass BH candidates have a mass around $10 M_{\odot}$. If the stellar companion is a few solar masses, the BH cannot significantly change its mass and spin angular momentum even swallowing the whole star. If the stellar companion is heavy, its lifetime is too short: even if the $\mathrm{BH}$ accretes at the Eddington rate, there is not the time to transfer the necessary amount of matter to significantly spin the $\mathrm{BH}$ up. In the end, a $\mathrm{BH}$ cannot swallow more than a few $M_{\odot}$ from the companion star, and for a $10 M_{\odot}$ object this is not enough to significantly change its spin parameter $a_{*}[7]$.

$\mathrm{BH}$ binaries can be grouped into two classes. Low-mass $\mathrm{X}$-ray binaries are systems in which the stellar companion is not more than a few solar masses $\left(\lesssim 3 M_{\odot}\right)$ and the mass transfer occurs for Roche lobe overflow. These systems are transient X-ray sources because the mass transfer is not continuous. High-mass X-ray binaries are systems in which the stellar companion is massive $\left(\gtrsim 10 M_{\odot}\right)$ and the mass transfer from the companion star to the $\mathrm{BH}$ is due to the wind of the former. These systems are persistent X-ray sources. If

\footnotetext{
1 Throughout the paper, we use units in which $G_{\mathrm{N}}=c=1$.
} 
the BH spin is mainly natal, its value should be explained by studying the gravitational collapse of massive stars. While there are still uncertainties in the angular momentum transport mechanisms of the progenitors of stellar-mass BHs, it is widely accepted that the gravitational collapse of a massive star with solar metallicity cannot create fast-rotating remnants $[9,10]$. The birth spin of these BHs is expected to be very low (see e.g. [8] and references therein). However, this is not what we observe. Assuming the Kerr metric, BH spin measurements show that some of these objects have a spin parameter close to 1 . In the case of low-mass X-ray binaries, the BH candidate in GRS $1915+105$ has $a_{*}>0.98$ [11] and $M=12.4 \pm 2.0 M_{\odot}[12]$, while the stellar-companion's mass is $M=0.52 \pm 0.41 M_{\odot}$. In the case of high-mass X-ray binaries, the BH candidate in Cygnus X-1 has $a_{*}>0.98[13,14]$ and $M=14.8 \pm 1.0 M_{\odot}$, while the stellar wind from the companion is not an efficient mechanism to transfer mass. Both spin constraints are at $3 \sigma$. While BHs in low- and high-mass X-ray binaries form in different environments, in both cases the origin of spin values so high is puzzling: the birth spin is expected to be low and accretion can spin a $\mathrm{BH}$ up only by transferring a significant amount of matter.

In this paper, we show that current spin measurements can easily be explained if $\mathrm{BH}$ candidates in X-ray binaries are not the Kerr BHs of general relativity. In particular, an initially non-rotating $\mathrm{BH}$ can look like a fast-rotating Kerr BH after accreting a small amount of matter $\left(\sim 2 M_{\odot}\right)$ if it is more prolate than a Kerr BH. Strictly speaking, this does not necessary mean that the $\mathrm{BH}$ must be prolate, but simply that it must be less oblate than the Kerr one. Here the key point is the innermost stable circular orbit (ISCO), which depends on the background metric. A BH more prolate than a Kerr one can look like a very fast-rotating Kerr BH when its spin parameter is much lower, which can be acquired after accreting a modest amount of mass. While the scenario is speculative and requires new physics, it is not in contradiction with any observation or theoretical argument $[15,16]$, and it provides a simple explanation to current spin measurements.

\section{Kerr black holes}

Accretion process from a thin disk is an efficient mechanism to spin a BH up. The inner edge of the disk is at the ISCO radius, as supported by observations [17]. The accreting gas moves on nearly geodesic circular orbits on the equatorial plane. As the gas loses energy and angular momentum, it first approaches the ISCO radius and then quickly plunges onto the BH. The mass and spin angular momentum of the $\mathrm{BH}$ change as

$$
M \rightarrow M+\delta M, \quad J \rightarrow J+\delta J,
$$

where $\delta M$ and $\delta J$ are the mass and the angular momentum carried by the gas

$\delta M=E_{\mathrm{ISCO}} \delta m, \quad \delta J=L_{\mathrm{ISCO}} \delta m$,

$E_{\mathrm{ISCO}}$ and $L_{\mathrm{ISCO}}$ are, respectively, the specific energy and the specific angular momentum of the gas at the ISCO radius, while $\delta m$ is the gas rest mass. With this set-up, one finds the well-known equation of the spin evolution [18]

$\frac{\mathrm{d} a_{*}}{\mathrm{~d} \ln M}=\frac{1}{M} \frac{L_{\mathrm{ISCO}}}{E_{\mathrm{ISCO}}}-2 a_{*}$.

In the case of the Kerr metric, it is possible to integrate Eq. (3) and find an analytic expression for the spin parameter $a_{*}$ as a function of the BH mass $M[19]$

$$
a_{*}= \begin{cases}\sqrt{\frac{2}{3}} \frac{M_{0}}{M}\left[4-\sqrt{18 \frac{M_{0}^{2}}{M^{2}}-2}\right] & \text { if } M \leq \sqrt{6} M_{0}, \\ 1 & \text { if } M>\sqrt{6} M_{0},\end{cases}
$$

assuming an initially non-rotating $\mathrm{BH}$ with mass $M_{0}$. The equilibrium value of the spin parameter is 1 and requires that the $\mathrm{BH}$ has increased its mass by a factor $\sqrt{6} \approx 2.4$. If we include the effect of the radiation emitted by the disk and captured by the $\mathrm{BH}$, we find that the equilibrium spin parameter is about 0.998, the so-called Thorne limit [18], since radiation with angular momentum opposite to the $\mathrm{BH}$ spin has larger capture cross section.

The left panel in Fig. 1 shows the evolution of $a_{*}$ as a function of the accreted mass for some values of the initial $\mathrm{BH}$ mass. An initially non-rotating $\mathrm{BH}$ has to double its original mass to get $a_{*}=0.98$. If a BH in an X-ray binary cannot strip more than a few solar masses from the stellar companion, only for low-mass BHs with $M \approx 3 M_{\odot}$ it may be possible to get $a_{*}=0.98$. An initially low value of the spin parameter does not help very much, since the evolution of the spin parameter is faster at the beginning and slower when the spin is higher. As the birth spin of BHs is expected to be low, we do not understand why we observe some fast-rotating BHs like GRS 1915+105. This one should have been born with a mass of $\sim 6 M_{\odot}$ and have accreted a similar amount of matter from the stellar companion, which seems to be unlikely. The right panel in Fig. 1 shows the evolution of the same systems in terms of the radiative efficiency $\eta=1-E_{\text {ISCO }}$. A Kerr BH with $a_{*}=0.98$ has a radiative efficiency $\eta=0.234$.

\section{Non-Kerr black holes}

If $\mathrm{BH}$ candidates in X-ray binaries were not the Kerr BHs of general relativity, current spin measurements would be wrong (because obtained assuming the Kerr metric) and the 


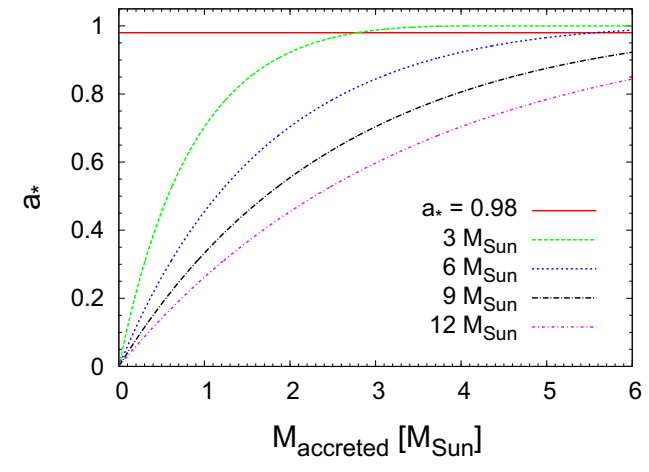

Fig. 1 Kerr BHs. Evolution of the spin parameter $a_{*}$ (left panel) and of the radiative efficiency $\eta=1-E_{\text {ISCO }}$ (right panel) as a function of the amount of matter accreted onto an initially non-rotating $\mathrm{BH}$ for four different initial $\mathrm{BH}$ masses $\left(3 M_{\odot}, 6 M_{\odot}, 9 M_{\odot}\right.$, and $\left.12 M_{\odot}\right)$. The

evolution of the spin parameter as a result of mass transfer from the stellar companion would be different. A number of studies have clearly shown that there is a fundamental degeneracy between the spin and possible deviations from the Kerr solution, with the result that a non-Kerr BH may be interpreted as a Kerr BH with a different value of $a_{*}$ [2023]. The main technique to estimate the spin parameter of stellar-mass BH candidates is the so-called continuum-fitting method, namely the study of the thermal spectrum of thin disks $[5,6]$. As a first crude approximation, the approach measures the radiative efficiency $\eta$ [24], which is then translated into a spin measurement under the assumption of the Kerr background, exploiting the fact there is a one-to-one correspondence between $\eta$ and $a_{*}$. It turns out that BHs more prolate than the Kerr ones have a higher radiative efficiency for a lower value of $a_{*}$ and they may thus look like very fastrotating Kerr BHs after acquiring a relatively small amount of mass from the stellar companion. The result is very general, but it is useful to see this with some specific example.

As a first case, we consider the Johannsen-Psaltis metric [25]. Observational constraints on this metric from stellar-mass BH candidates are discussed in [24]. In BoyerLindquist coordinates, the line element reads [25]

$$
\begin{aligned}
\mathrm{d} s^{2}= & -\left(1-\frac{2 M r}{\Sigma}\right)(1+h) \mathrm{d} t^{2} \\
& +\frac{\Sigma(1+h)}{\Delta+a^{2} h \sin ^{2} \theta} \mathrm{d} r^{2}+\Sigma \mathrm{d} \theta^{2} \\
& -\frac{4 a M r \sin ^{2} \theta}{\Sigma}(1+h) \mathrm{d} t \mathrm{~d} \phi \\
& +\left[\sin ^{2} \theta\left(r^{2}+a^{2}+\frac{2 a^{2} M r \sin ^{2} \theta}{\Sigma}\right)\right. \\
& \left.+\frac{a^{2}(\Sigma+2 M r) \sin ^{4} \theta}{\Sigma} h\right] \mathrm{d} \phi^{2},
\end{aligned}
$$

where $a=J / M, \Sigma=r^{2}+a^{2} \cos ^{2} \theta$, and $\Delta=r^{2}-2 M r+$ $a^{2} . h$ introduces deviations from the Kerr background and in

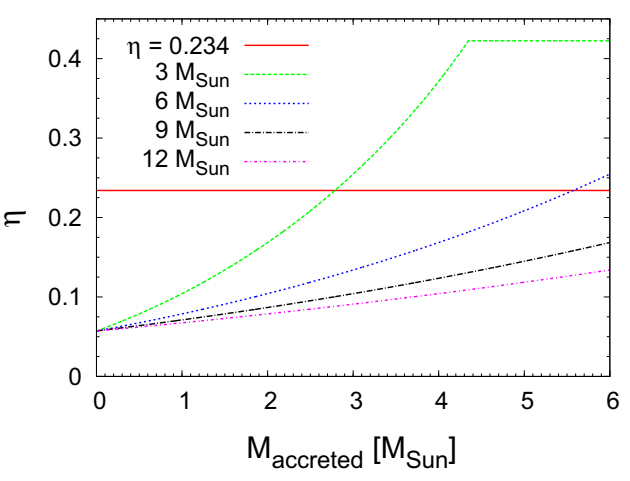

horizontal red solid lines indicate the spin parameter $a_{*}=0.98$ and the corresponding radiative efficiency $\eta=0.234$. See the text for more details

its simplest version it is given by

$h=\frac{\epsilon_{3} r M^{3}}{\Sigma^{2}}$,

where $\epsilon_{3}$ is an unknown parameter that quantifies possible deviations from the Kerr solution. Johannsen-Psaltis BHs are more prolate (oblate) than their Kerr cousins with the same spin parameter when $\epsilon_{3}>0\left(\epsilon_{3}<0\right)$ [26].

The Johannsen-Psaltis metric is a phenomenological metric and does not describe any known solution of spinning BHs in modified gravity. It can be used as a toy model to described non-Kerr BHs assuming that particles follow the geodesics of its spacetime, namely that it can be obtained as a solution (or approximated solution) of some metric theory of gravity. In this case, it is possible to study the evolution of the spin parameter of these non-Kerr objects [27-29]. The master equation is still (3), but $E_{\mathrm{ISCO}}$ and $L_{\mathrm{ISCO}}$ are different because they are determined by the background metric.

In the original proposal of Ref. [25], $\epsilon_{3}$ is a phenomenological parameter quantifying possible deviations from the Kerr geometry. In presence of an underlying theory, it may be related to some coupling parameter in the modified action and thus be a constant of the theory. Figure 2 shows the evolution of the spin parameter $a_{*}$ and of the radiative efficiency $\eta$ of these Johannsen-Psaltis BHs for $\epsilon_{3}=4$. Another possible scenario is that non-rotating BHs are spherically symmetric and that rotation makes the object more and more deformed. For instance, Kerr BHs have a mass-quadrupole moment given by $Q=-a_{*}^{2} M^{3}: Q=0$ in the non-rotating case and the $\mathrm{BH}$ is more and more oblate as the spin parameter increases. The mass-quadrupole moment of neutron stars can be approximated by $Q=-(1+\chi) a_{*}^{2} M^{3}$, where $\chi \sim 1-$ 10 is a parameter that mainly depends on the matter equation of states and at some level on the mass $M$ [30]. Since $\chi>0$, neutron stars are more oblate than Kerr BHs with the same spin parameter $a_{*}$. It is thus possible that $\epsilon_{3}$ depends on the 

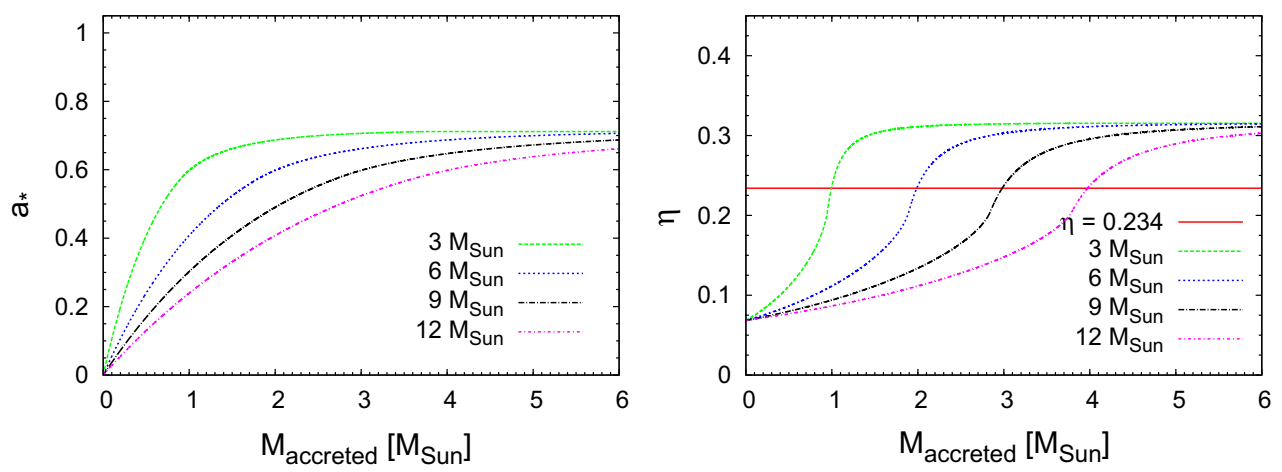

Fig. 2 Non-Kerr BHs. As in Fig. 1, but in the case of Johannsen-Psaltis BHs with $\epsilon_{3}=4$. The key point is that here $\eta$ can become high after a modest mass transfer. See the text for more details
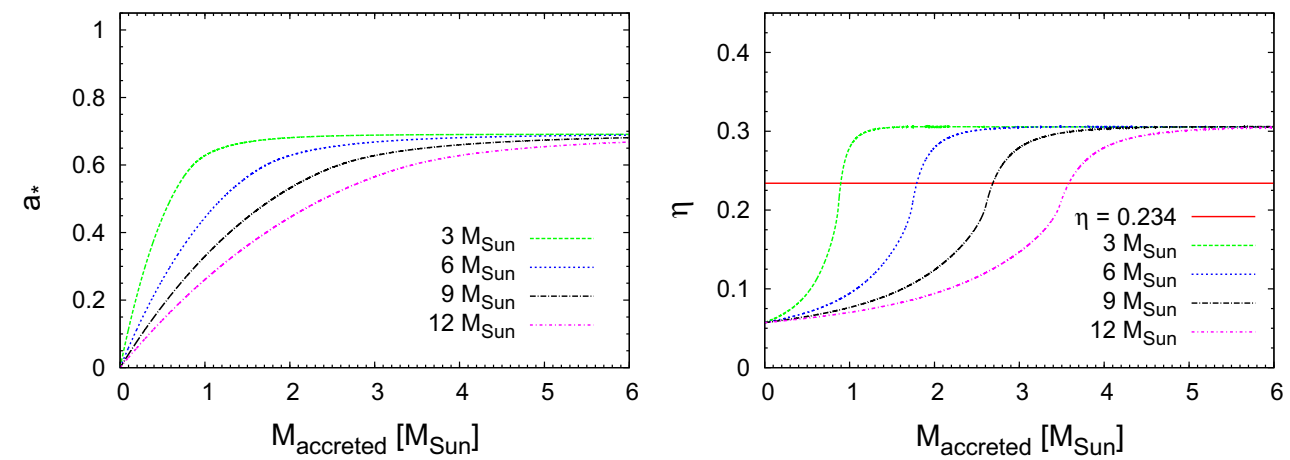

Fig. 3 Non-Kerr BHs. As in Fig. 1, but in the case of Johannsen-Psaltis BHs with $\epsilon_{3}=10 a_{*}^{2}$. See the text for more details

spin parameter, and the simplest case is that it is proportional to $a_{*}^{2}$, say $\epsilon_{3}=k a_{*}^{2}$; Fig. 3 shows the evolution of the spin parameter $a_{*}$ and of the radiative efficiency $\eta$ of these Johannsen-Psaltis BHs for $k=10$. In both scenarios, we find two remarkable features of these BHs:

(i) the equilibrium spin parameter is much lower than 1, and

(ii) an initially non-rotating $\mathrm{BH}$ reaches a high radiative efficiency very quickly, after a modest amount of mass transfer. This is a preliminary indication that mass accretion onto a non-Kerr BH may explain the observation of fast-rotating Kerr BHs in X-ray binaries.

The argument of the radiative efficiency can be used only for a preliminary estimate and deviations are more important for high values of $\eta$, mainly because in the spin measurement from the disk's thermal spectrum there is a correlation between the estimate of $a_{*}$ and the mass accretion rate [24]. Reference [24] reports the current constraints on $a_{*}$ and $\epsilon_{3}$ of the 10 stellar-mass BH candidates with a spin measurement. The left panel in Fig. 4 shows the evolution of some Johannsen-Psaltis BHs on the plane $\left(a_{*}, \epsilon_{3}\right)$ as well as the boundary of the allowed region (red solid line) for the $\mathrm{BH}$ in GRS 1915+105 (the allowed region is the area inside the line). With the ansatz $\epsilon_{3}=k a_{*}^{2}$, we can see that $k$ cannot be too high. For $k=0$, we recover the standard Kerr met- ric and a too large amount of matter is necessary to spin up a $\mathrm{BH}$. For $k=10$, an initially non-rotating $\mathrm{BH}$ with a mass $M=9.4 M_{\odot}$ enters the allowed region after accreting 3.0 $M_{\odot}$. The final mass of $12.4 M_{\odot}$ would correspond to the mass measurement of the BH in GRS 1915+105. For $k=5$, an initially non-rotating $\mathrm{BH}$ with a mass $M=9.1 M_{\odot}$ enters the allowed region after accreting $3.3 M_{\odot}$. In the case of the BH in Cygnus X-1, we can obtain similar results.

The case of the Johannsen-Psaltis BHs is just an example. The result is very general, in the sense that BHs more prolate than the Kerr ones can look like fast-rotating Kerr BHs after accreting a modest amount of mass. The necessary amount of mass is clearly related to the specific non-Kerr model. For instance, the Cardoso-Pani-Rico BHs [31] are a simple generalization of the Johannsen-Psaltis ones. In this case, there is no systematic study of current constraints for all the $\mathrm{BH}$ candidates, but the case of Cygnus X-1 was analyzed in [32]. In Boyer-Lindquist coordinates, the line element reads [31]

$$
\begin{aligned}
\mathrm{d} s^{2}=- & \left(1-\frac{2 M r}{\Sigma}\right)\left(1+h^{t}\right) \mathrm{d} t^{2}-2 a \sin ^{2} \theta \\
& \times\left[\sqrt{\left(1+h^{t}\right)\left(1+h^{r}\right)}-\left(1-\frac{2 M r}{\Sigma}\right)\left(1+h^{t}\right)\right] \mathrm{d} t \mathrm{~d} \phi \\
& +\frac{\Sigma\left(1+h^{r}\right)}{\Delta+h^{r} a^{2} \sin ^{2} \theta} \mathrm{d} r^{2}+\Sigma \mathrm{d} \theta^{2}
\end{aligned}
$$




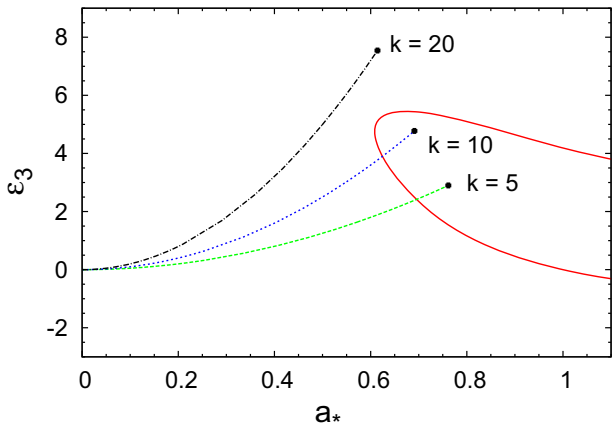

Fig. 4 Left panel: evolution of Johannsen-Psaltis BHs with $\epsilon_{3}=k a_{*}^{2}$ on the plane $\left(a_{*}, \epsilon_{3}\right)$ as a result of the accretion process. The black dots indicate the equilibrium configurations. The red solid line is the boundary of the allowed region of the BH candidate in GRS 1915+105 (see Ref. [24]). For $k=10$, an initially non-rotating BH with a mass $M=9.4 M_{\odot}$ enters the allowed region after a mass transfer

$$
\begin{aligned}
& +\sin ^{2} \theta\left\{\Sigma+a^{2} \sin ^{2} \theta\left[2 \sqrt{\left(1+h^{t}\right)\left(1+h^{r}\right)}\right.\right. \\
& \left.\left.-\left(1-\frac{2 M r}{\Sigma}\right)\left(1+h^{t}\right)\right]\right\} \mathrm{d} \phi^{2}
\end{aligned}
$$

where, in the simplest version, $h^{t}$ and $h^{r}$ are

$h^{t}=\epsilon_{3}^{t} \frac{r M^{3}}{\Sigma^{2}}, \quad h^{r}=\epsilon_{3}^{r} \frac{r M^{3}}{\Sigma^{2}}$.

As shown in Ref. [32], current observations can strongly constrain $\epsilon_{3}^{t}$, while the bounds are very weak for $\epsilon_{3}^{r}$. We can thus consider the case $\epsilon_{3}^{t}=0$ and $\epsilon_{3}^{r}=k a_{*}^{2}$ and compute the amount of mass transfer necessary to explain the $\mathrm{BH}$ spin in Cygnus X-1. The calculations are reported in the right panel in Fig. 4. There are three cases, respectively for $k=10,20$, and 30 . The area between the two red solid curves is the region allowed by observations for Cygnus X-1 and obtained in Ref. [32]. The mass of the BH in Cygnus X-1 is $M=14.8 \pm 1.0 M_{\odot}$. For $k=30$, an initially non-rotating BH with a mass $M=12.4 M_{\odot}$ enters the allowed region after a mass transfer $M_{\text {accreted }}=2.4 M_{\odot}$. For a lower value of $k$ a larger amount of mass is necessary. For instance, in the case $k=10$ an initially non-rotating $\mathrm{BH}$ with a mass $M=11.6 M_{\odot}$ enters the allowed region after a mass transfer $M_{\text {accreted }}=3.2 M_{\odot}$.

It should not be difficult to find more efficient nonKerr models, namely BHs that can look like very-fast rotating Kerr BHs after accreting a smaller amount of matter. However, it has to be noted that the Johannsen-Psaltis and Cardoso-Pani-Rico spacetimes are phenomenological metrics to parameterize generic deviations from the Kerr solution. Some spinning BH solutions in well-motivated alternative theories of gravity are known [33-36]. Generally speaking, if we consider a specific alternative theory of gravity,

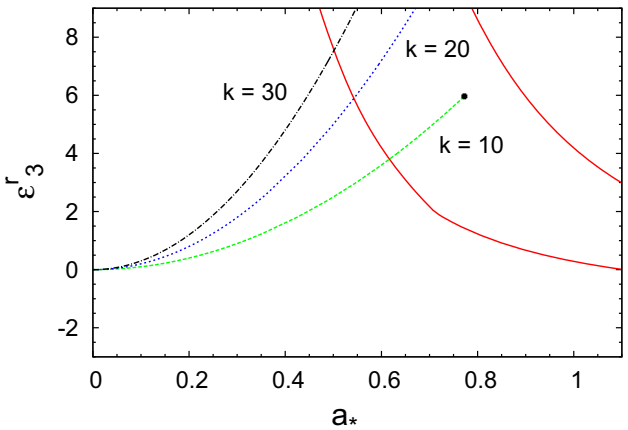

$M_{\text {accreted }}=3.0 M_{\odot}$. Right panel: evolution of Cardoso-Pani-Rico BHs with $\epsilon_{3}^{r}=k a_{*}^{2}$ on the plane $\left(a_{*}, \epsilon_{3}^{r}\right)$ as a result of the accretion process. The red solid line is the boundary of the allowed region of the BH candidate in Cygnus X-1 (see Ref. [32]). For $k=30$, an initially non-rotating $\mathrm{BH}$ with a mass $M=12.4 M_{\odot}$ enters the allowed region after a mass transfer $M_{\text {accreted }}=2.4 M_{\odot}$. See the text for more details

it is possible that its $\mathrm{BH}$ solutions are not sufficiently more prolate than Kerr for any choice of the parameters of the theory. Moreover, the deformation parameters in the JohannsenPsaltis and Cardoso-Pani-Rico spacetimes are only constrained by observations sensitive to the metric (assuming geodesic motion), not by the field equations of the theory (which are not given). If we have a theory, there may be independent bounds coming from the field equations (e.g. emission of gravitational waves from a binary pulsar). After satisfying these constraints, it is not obvious that their BHs can do the job proposed in the present paper. Every theory is different and it should be analyzed by itself. There are also scenarios like the one suggested in [37,38], in which general relativity holds up to quite strong gravitational fields, but BHs do not have the usual properties (even the concept of metric breaks down on the surface of these objects) and, in many aspects, they behave like compact stars made of exotic matter. In this case, constraints can only be obtained by $\mathrm{BH}$ observational data and the current bounds on possible deviations from the Kerr solution are weak [24].

\section{Concluding remarks}

In this paper, we showed that current spin measurements of BHs in X-ray binaries may easily be explained if these objects are more prolate than the predictions of general relativity. The point is that similar objects can look like very fast-rotating Kerr BHs with a lower value of the spin parameter, which can be acquired after a modest mass transfer from the stellar companion. In the case of Kerr BHs, the required amount of mass stripped from the stellar companion is too high and therefore it is not understood the origin of so high spins for some BHs in X-ray binaries. It is at least intriguing that even 
other observations seem to require that stellar-mass BH candidates are more prolate than the Kerr ones, namely the power of steady jets [39] and quasi-periodic oscillations [40,41]. At this stage, the proposal that BH candidates are not the Kerr $\mathrm{BHs}$ of general relativity is a very speculative possibility, but it is not in contradiction with any observation. It is probably difficult to test this scenario with a more detailed study of the origin of the $\mathrm{BH}$ spin, but future observational facilities will be hopefully able to test the Kerr nature of astrophysical $\mathrm{BH}$ candidates [42-44].

Acknowledgments This work was supported by the NSFC grant No. 11305038, the Shanghai Municipal Education Commission grant for Innovative Programs No. 14ZZ001, the Thousand Young Talents Program, and Fudan University.

Open Access This article is distributed under the terms of the Creative Commons Attribution License which permits any use, distribution, and reproduction in any medium, provided the original author(s) and the source are credited.

Funded by $\mathrm{SCOAP}^{3}$ / License Version CC BY 4.0.

\section{References}

1. R.A. Remillard, J.E. McClintock, Ann. Rev. Astron. Astrophys. 44, 49 (2006). arXiv:astro-ph/0606352

2. C.E. Rhoades Jr, R. Ruffini, Phys. Rev. Lett. 32, 324 (1974)

3. C. Bambi, K. Freese, Phys. Rev. D 79, 043002 (2009). arXiv:0812.1328 [astro-ph]

4. C. Bambi, D. Malafarina, N. Tsukamoto, Phys. Rev. D 89, 127302 (2014). arXiv:1406.2181 [gr-qc]

5. J.E. McClintock, R. Narayan, S.W. Davis et al., Class. Quant. Grav. 28, 114009 (2011). arXiv:1101.0811 [astro-ph.HE]

6. J. E. McClintock, R. Narayan, J. F. Steiner, doi:10.1007/ s11214-013-0003-9. arXiv:1303.1583 [astro-ph.HE]

7. A.R. King, U. Kolb, Mon. Not. R. Astron. Soc. 305, 654 (1999). arXiv:astro-ph/9901296

8. T. Fragos, J. E. McClintock. arXiv:1408.2661 [astro-ph.HE]

9. S.E. Woosley, J.S. Bloom, Ann. Rev. Astron. Astrophys. 44, 507 (2006). arXiv:astro-ph/0609142

10. S.C. Yoon, N. Langer, C. Norman, Astron. Astrophys. 460, 199 (2006). arXiv:astro-ph/0606637

11. J.E. McClintock, R. Shafee, R. Narayan et al., Astrophys. J. 652, 518 (2006). arXiv:astro-ph/0606076

12. M. J. Reid, J. E. McClintock, J. F. Steiner et al. arXiv:1409.2453 [astro-ph.GA]

13. L. Gou, J.E. McClintock, M.J. Reid et al., Astrophys. J. 742, 85 (2011). arXiv:1106.3690 [astro-ph.HE]

14. L. Gou, J.E. McClintock, R.A. Remillard et al., Astrophys. J. 790, 29 (2014). arXiv:1308.4760 [astro-ph.HE]
15. C. Bambi, Mod. Phys. Lett. A 26, 2453 (2011). arXiv: 1109.4256 [gr-qc]

16. C. Bambi, Astron. Rev. 8, 4 (2013). arXiv:1301.0361 [gr-qc]

17. J.F. Steiner, J.E. McClintock, R.A. Remillard et al., Astrophys. J. 718, L117 (2010). arXiv:1006.5729 [astro-ph.HE]

18. K.S. Thorne, Astrophys. J. 191, 507 (1974)

19. J.M. Bardeen, Nature 226, 64 (1970)

20. C. Bambi, E. Barausse, Astrophys. J. 731, 121 (2011). arXiv:1012.2007 [gr-qc]

21. H. Krawczynski, Astrophys. J. 754, 133 (2012). arXiv:1205.7063 [gr-qc]

22. C. Bambi, Phys. Rev. D 87, 023007 (2013). arXiv:1211.2513 [gr-qc]

23. C. Bambi, JCAP 1308, 055 (2013). arXiv:1305.5409 [gr-qc]

24. L. Kong, Z. Li, C. Bambi, Astrophys. J. 797, 78 (2014). arXiv:1405.1508 [gr-qc]

25. T. Johannsen, D. Psaltis, Phys. Rev. D 83, 124015 (2011). arXiv:1105.3191 [gr-qc]

26. C. Bambi, L. Modesto, Phys. Lett. B 706, 13 (2011). arXiv:1107.4337 [gr-qc]

27. C. Bambi, Europhys. Lett. 94, 50002 (2011). arXiv:1101.1364 [gr-qc]

28. C. Bambi, JCAP 1105, 009 (2011). arXiv:1103.5135 [gr-qc]

29. C. Bambi, Phys. Lett. B 705, 5 (2011). arXiv:1110.0687 [gr-qc]

30. W. G. Laarakkers, E. Poisson, Astrophys. J. 512, 282 (1999). arXiv:gr-qc/9709033

31. V. Cardoso, P. Pani, J. Rico, Phys. Rev. D 89(6), 064007 (2014). arXiv:1401.0528 [gr-qc]

32. C. Bambi, Phys. Rev. D 90, 047503 (2014). arXiv:1408.0690 [grqc]

33. B. Kleihaus, J. Kunz, E. Radu, Phys. Rev. Lett. 106, 151104 (2011). arXiv:1101.2868 [gr-qc]

34. P. Pani, C.F.B. Macedo, L.C.B. Crispino, V. Cardoso, Phys. Rev. D 84, 087501 (2011). arXiv:1109.3996 [gr-qc]

35. K. Yagi, N. Yunes, T. Tanaka, Phys. Rev. D 86, 044037 (2012) (erratum-ibid. D 89(4), 049902, 2014). arXiv:1206.6130 [gr-qc]

36. D. Ayzenberg, N. Yunes, Phys. Rev. D 90, 044066 (2014). arXiv:1405.2133 [gr-qc]

37. G. Dvali, C. Gomez, Fortsch. Phys. 61, 742 (2013). arXiv:1112.3359 [hep-th]

38. G. Dvali, C. Gomez, Phys. Lett. B 719, 419 (2013). arXiv:1203.6575 [hep-th]

39. C. Bambi, Phys. Rev. D 86, 123013 (2012). arXiv:1204.6395 [grqc]

40. C. Bambi, JCAP 1209, 014 (2012). arXiv:1205.6348 [gr-qc]

41. C. Bambi, arXiv:1312.2228 [gr-qc]

42. Z. Li, L. Kong, C. Bambi, Astrophys. J. 787, 152 (2014). arXiv:1401.1282 [gr-qc]

43. N. Tsukamoto, Z. Li, C. Bambi, JCAP 1406, 043 (2014). arXiv:1403.0371 [gr-qc]

44. J. Jiang, C. Bambi, J. F. Steiner, arXiv:1406.5677 [gr-qc] 